\title{
MAC LAYER PERFORMANCE: STUDY AND ANALYSIS FOR DIFFERENT MOBILITY CONDITIONS IN WSN.
}

\author{
Manjusha Pandey ${ }^{1}$, Shekhar Verma ${ }^{2}$ \\ Indian Institute of Information Technology Allahabad, India \\ \{rs58 ${ }^{1}$, sverma ${ }^{2}$ eiitita.ac.in
}

\begin{abstract}
Wireless sensor networks are the most challenging networks for communication because of its resource constrained nature and the dynamical nature of network topographic anatomy. A lot of research is being going on in the diverse parts of the world for optimum utilization of communication resources in these special types of ad hoc networks. The utility and application domain of sensor networks ranges from commercial, public safety applications and military sector to be the most important ones. The magnificent challenges to the routing algorithms employed in such type of networks are due to the mercurial size of the network and its expandable topology that is quite dynamic in nature. The present paper offers a comparison and analysis of the packet drop at the MAC layer for different routing protocols under an experimental setup having different mobility condition based scenarios of the wireless sensor network application. The comparative study may have also an impact on the improvement of MAC layer performance for different simulation times of the experimental setup considering two of reactive as well as proactive protocols that are most widely used routing protocol in wireless sensor networks. Wireless sensor network application under consideration for the experimental is the battle field monitoring wireless sensor network and the comparative study has been performed for four different mobility patterns described as four different scenarios in the considered experimental application of wireless sensor networks.
\end{abstract}

The sensor network simulative electronic deception architecture used is for the battle field monitoring application of wireless sensor networks. The application provides support for sensing capabilities within the network nodes called as UGS (Unattended Ground Sensors).Mobile nodes gather data from battle field and direct it to the base station via mobile UGV (Unmanned Ground Vehicles).The performance of the MAC Layer varies with the different average jitter values for different simulation times in the network. Power usage model has been used to reliably represent an actual sensor hardware and sensor network oriented traffic pattern.

\section{KEYWORDS}

Medium Access Control Protocol, Wireless Sensor Networks, Packet Drop .AODV, OLSR, DYMO, LANMAR

\section{INTRODUCTION}

Wireless sensor networking is an emerging technology that has potential usage in environment monitoring, defense, smart spaces, scientific application, medical systems and robotic exploration, target tracking, intrusion detection, wildlife habitat monitoring, climate control and disaster management etc. Wireless sensor network (WSN) consist of one or more batteryoperated sensor devices with embedded processor, small memory and low power radio. Low

DOI : 10.5121/ijwmn.2011.3609 
power capacities of sensor node results in limited coverage and communication range for sensor nodes compared to other mobile devices. Hence, to successfully cover the target area, sensor networks are composed of large number of nodes. Nodes in wireless sensor network coordinate to perform a common task.

Medium access control (MAC) protocol in wireless networks has an important role to enable the successful operation of the network. One fundamental task of the MAC protocol is to avoid collisions so that two interfering nodes do not transmit at the same time. There are many MAC protocols that have been developed for wireless networks. Typical examples include the time division multiple access (TDMA), code division multiple access (CDMA), and contentionbased protocols like IEEE 802.11.In a wireless network, controlling when to send a packet and when to listen for a packet are two most important operations to be performed by the medium access layer. In general, idly waiting wastes huge amounts of energy during communication. Medium access control protocol deals with when and how to access medium by a node, and how to transfer the data safely when there is more than one node accessing a single wireless channel simultaneously. MAC layer is a part of DLC (data link layer) which is divided into MAC and LLC (Logical link layer) sub layers. The main task of LLC is Error and Flow Control. MAC layer resolves contentions in a multi-access wireless environment. Problems in medium access are influenced by a number of attributes and trade off's like - Collision Avoidance, Energy Efficiency, Scalability and adaptivity, Channel Utilization, Latency, Throughput and Fairness.

Because of the mobility of nodes, there are certain characteristics that are only applicable to adhoc networks. Some of these key characteristics are bandwidth constrained links, dynamic network topologies, and energy constrained operations. In the real-time applications, and realtime data, the ad hoc networks allow for Quality of Service (QoS) in terms of delay, bandwidth, as well as packet loss. This network does not have defined routers and routes. All nodes have capability of moving, may work as routers, and can be connected in an arbitrary manner. Functioning as routers, these nodes discover as well as maintain routes to other node within the network. The nodes move around randomly, thus making the network topology dynamic in nature. So it is important for the routing protocols to be adaptive and have the ability to maintain routes in spite of dynamic network topology. These networks have drew in a lot of attention throughout the past several years because of increased demand for ubiquitous connectivity and emergence of new communication scenarios such as sensor networks Some critical areas of applications of these networks are in the fields of military and civilian application such as communication in the battle field, disaster management, vehicular movement or communication in traffic management and scientific exploration etc. In all these applications, group communication is more important.

The present research effort concentrates on the sensor network simulator architecture that furnishes support for sensing potentialities in network nodes, existent sensor hardware and sensor network orienting traffic model. We have contemplated sensor network models in the various circumstance of network simulation and this is the exclusive work to our cognition that compares the routing mechanisms for detailed models on the operation of sensor networks [2]. Four different mobility condition based scenarios illustrate the comparative analysis of using accurate and representative wireless sensor network models.

\section{State OF ART}

In wireless ad hoc networks [9] out of numerous views to be taken into thoughtfulness one of the most significant is that of the effective energy management with the additional goal of prolonged connectivity of the network and increased lifetime of the network. These constraints are particularly true of sensor networks. In these networks the nodes are usually battery powered and left unattended after deployment. The routing algorithms designed for these 
networks need to monitor the energy of nodes and route packets accordingly. Considering the work done in the field of comparison and analysis, the analysis has been done between the routing protocols evaluated based on quantitative and qualitative metrics [14]. But the analysis of a protocols performance for exhaustive variations in simulation time of the same network and for different application scenarios has not been proposed and performed yet. A great deal of research in the domain of routing protocols in ad hoc networks has been done; AODV, DYMO, OLSR, LANMAR to mentioned a few.

OLSR [3] is a variation of traditional link state routing, modified for improved operation in ad hoc networks. The key feature of OLSR protocol is that it uses multipoint relays (MPRs) to reduce the overhead of network floods and size of link state updates. The OLSR protocol executes the hop by hop routing i.e. each node uses its most recent information to route a packet. States involved in the same are as neighbor sensing, multipoint relay station, MPR information declaration, routing table calculation.

LANMAR [12] aggregates the characteristics of Fisheye State Routing (FSR) [13] and Landmark routing. The fundamental novel characteristic is the role of landmarks for each set of nodes that move like a group (e.g., a team of co-workers at a convening or a tank battalion in the battleground) in order to subdue routing update operating expense. On the other hand, Ondemand routing protocols like AODV [5], DYMO [8] etc. are more dynamic. Instead of periodically updating the routing information, these protocols update routing information whenever a routing is required. This type of routing produces routes only when in demand by the source node and therefore, in general, the signaling overhead is reduced compared to proactive approaches of routing.

DYMO is meant for use by moving nodes in wireless, multi-hop networks. DYMO determines unicast amongst DYMO routers in the network in an on-demand manner, offering bettered convergence in dynamic topologies. The introductory procedures of the DYMO protocol are route finding (by route request and route reply) and route maintenance. It is an improvement to AODV and more comfortable to implement. In networks with a prominent number of routers, it is best suited for sparse traffic scenarios. In each DYMO router, minimal state routing is preserved and therefore it is relevant to memory constrained devices. The protocol is suitable for scalability. However, it is yet to be explored for its functionality.

\section{Mobility CONDITIONS AND IMPLEMENTATIONS}

This scenario under consideration for the present research effort demonstrates data collection from ground sensors using mobile vehicles for a battle field monitoring system. Sensors are randomly deployed in an observation region. The sensors constantly monitor any phenomena of interest in the area. The sensory information observed by each sensor is stored locally at the sensor. The mobile vehicles have short range communication to sensors and long distance communication to a remote site which is called fusion centre in this scenario. The sensors send their locally stored data packets to the vehicles which at any time are within their radio range. The vehicles then relay sensory data packets to fusion centre using long distance communication to that centre. The sensors which have CBR flows to fusion centre then are able to send their sensory data to the centre. The four different conditions for the scenario taken as mentioned above may be described as follows:

a) All nodes of the network are static. The UGS (unattended ground sensors) as well as the UGV (Unmanned ground station) are static while the fusion centre remains static in each and every condition in which the scenario has been implemented.

b) In the second condition of scenario implementation the UGS (unattended ground stations) are static while the UGV (unmanned ground station) are mobile. 
c) The third condition of scenario says the implementation of the UGS (unattended ground stations) is mobile while the UGV (unmanned ground station) are static.

d) The last condition of scenario implementation refers to situation when the UGS (unattended ground stations) are mobile as well as the UGV (unmanned ground station) are also mobile.

\section{Metric ANd Methodology OvervieW}

Advantages of MAC protocols for sensor networks include that the energy waste caused by idle listening is reduced by sleep schedules. Beside implementation simplicity, global time synchronization overhead may be prevented with sleep schedule announcements. There are also some disadvantages of MAC protocols for sensor network are, having a fixed duty cycle i.e. Active time is fixed. It is not optimal. If message rate is less energy is still wasted in idlelistening. Sleep and listen periods are predefined and constant which decreases the efficiency of the algorithm under variable traffic load. Long listening interval is expensive - Everyone stays awake unless somebody transmits .Time synchronization overhead even when network is idle.RTS/CTS and ACK overhead when sending data.

While traditional MAC protocols are designed to maximize packet throughput, minimize latency and provide fairness, protocol design for wireless sensor networks focuses on minimizing energy consumption. The application determines the requirements for the minimum through-put and maximum latency. Fairness is usually not an issue, since the nodes in a wireless sensor network are typically part of a single application and work together for a common purpose. The major sources of energy waste in a MAC protocol for wireless sensor networks are the following:

a) Collision: When a transmitted packet is corrupted it has to be discarded, and the followon retransmissions increase energy consumption.

b) Control Packet Overhead: Sending and receiving control packets consumes energy too, and less useful data packets can be transmitted.

c) Idle Listening: Listening to receive possible traffic that is not sent can consume extra energy.

d) Overhearing: Meaning that a node picks up packets that are destined to other nodes can unnecessarily consume energy.

The medium-access layer has two functions that impact packet delivery performance: arbitrating access to the channel, and (optionally) some simple form of error detection. In addition to factors that impact the physical layer, and hence the performance of medium-access, two factors affect the medium-access layer. First, the application workload (and, in the case of sensor networks, the sensed environment) determines the traffic generated by nodes and hence the efficacy of channel access. Second, the topology (or, equivalently, the spatial relationship between nodes) affects how many nodes might potentially contend for the channel at a given point in time. To understand packet delivery performance as observed at the MAC layer, we use the following general setup. We place sixty nodes in a somewhat ad-hoc fashion, but at densities that we expect of sensor network deployments. Each node periodically generates a message destined to one of its neighbors; the periodicity of this message generation defines an artificial workload. We then place this setup in three environments as before, and measure several aspects of packet delivery performance. 
The overall goal of our experiments was to compare and analyze the packets dropped ratio of the two considered reactive and proactive protocols for various application scenarios considered for the experimental simulations. Also the analysis has been done for routing protocol during the variations in the simulation time for the experimental setup. The protocols were carefully implemented according to its specifications. During the process of implementation of the AODV routing protocol and analyzing the results for each simulation runs, we discovered some modifications in the average jitter of the network for each simulation interval the network varied its performance, while carrying on to succeed to deliver data packets to their destinations. To measure these variations, our basic methodology was to apply to a simulated network a variety of, simulation intervals and different application scenarios implementing various mobility conditions that affect the routing protocols performance, and it's testing with each data packet originated by some sender whether the routing protocol can at that time route to the destination of that packet. We were not attempting to measure the protocols' performance on a particular workload taken from real life, but rather to measure the protocols' performance under a range of conditions.

\section{APPLiCation AND ANALYSIS}

The average delivery ratio decrements as channel error rate gains due to the increased packet loss error rate and this begins, reflecting the packet loss obtained both by increased congestion and due to packet loss at the MAC Layer. The packet loss rate at the MAC layer (between two routers, or between a router and a host) must be made very small in order to achieve better network routing protocol performance .It is the job of the MAC layer to achieve this condition of optimized data packet control.

The primary aspect of wireless communication performance of interest to us is packet delivery performance. More precisely, our primary measure of performance is packet loss rate (the fraction of packets that were transmitted within a time window, but not received) or its complement, the reception rate. There are many, many factors that govern the packet delivery performance in a wireless communication system: the environment, the network topology, the traffic patterns and, by extension, the actual physical phenomena that trigger node communication activity. It is difficult to isolate these phenomena in order to study the impact of different factors on packet delivery performance. Rather, we take a somewhat mechanistic view in this paper, and look at the packet delivery performance at two different layers in the networking stack: the physical layer and the medium-access layer

AODV routing protocol with the increase in the simulation time represents a steep variation from a high rate of packet loss for the highest simulation time having the highest average of 2700 packets lost to a low of 100 packets lost during the lowest simulation interval for which the application was implemented Though for the lesser variation in the simulation time the net packet loss at the MAC layer does not varies much. 
International Journal of Wireless \& Mobile Networks (IJWMN) Vol. 3, No. 6, December 2011

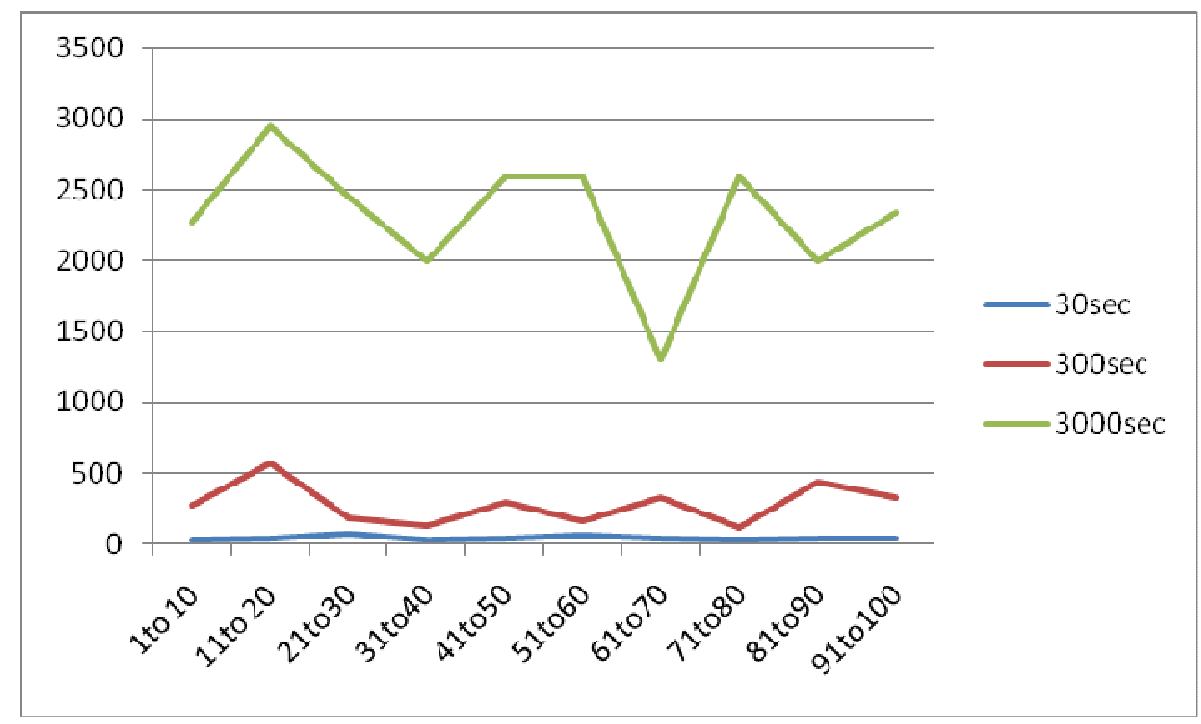

Figure 1. Packet Drop at MAC layer for AODV.

DYMO being an on demand reactive routing protocol like AODV still performs best for higher simulation time while the net packet loss presenting a minimum value of less than 100 packets loosed for the highest simulation time though with decrease in the simulation time the packet drop rate has increased thus making DYMO a suitable choice for longer period of simulation or network utilization in case of real life applications.

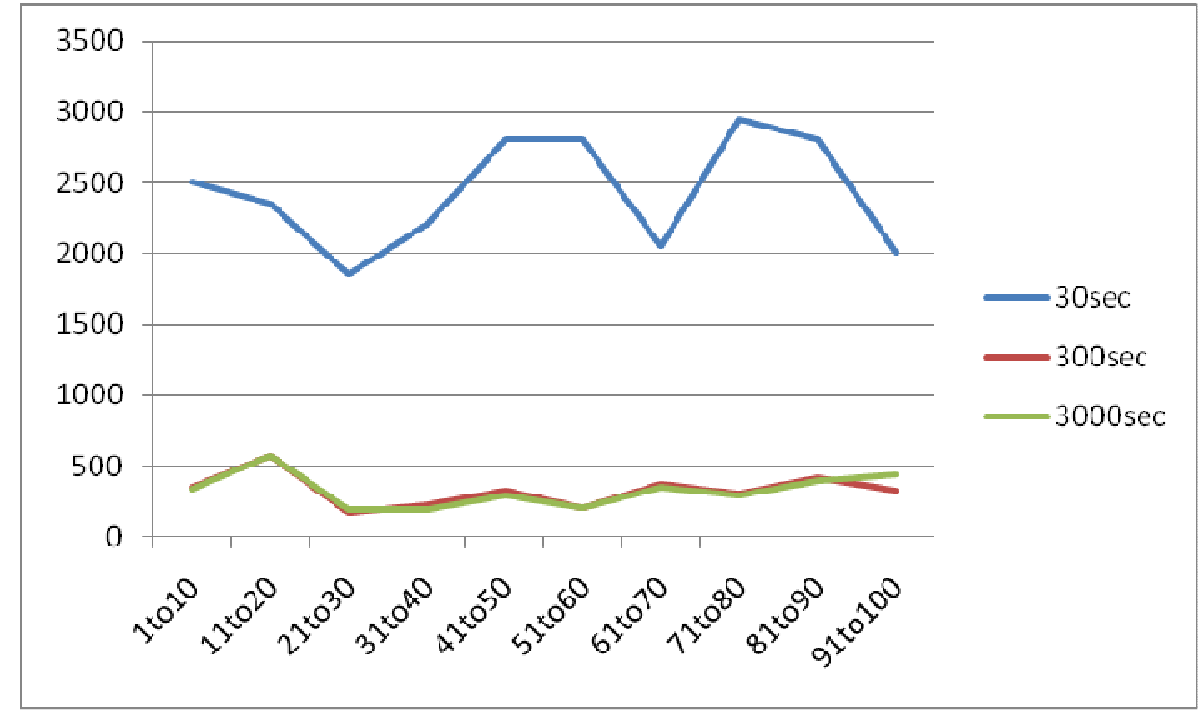

Figure 2. Packet Drop at MAC layer for DYMO.

OLSR uses the proactive methodology of routing techniques depending on the link states of the network. The results being analyzed show that this routing protocol produces best MAC layer performance in the case of smallest simulation time interval with the lowest number of packets dropped in that case being lesser than 50 packets dropped and the maximum packet drop was visible for the largest simulation time the highest value of packet dropped being 4500 . 
International Journal of Wireless \& Mobile Networks (IJWMN) Vol. 3, No. 6, December 2011

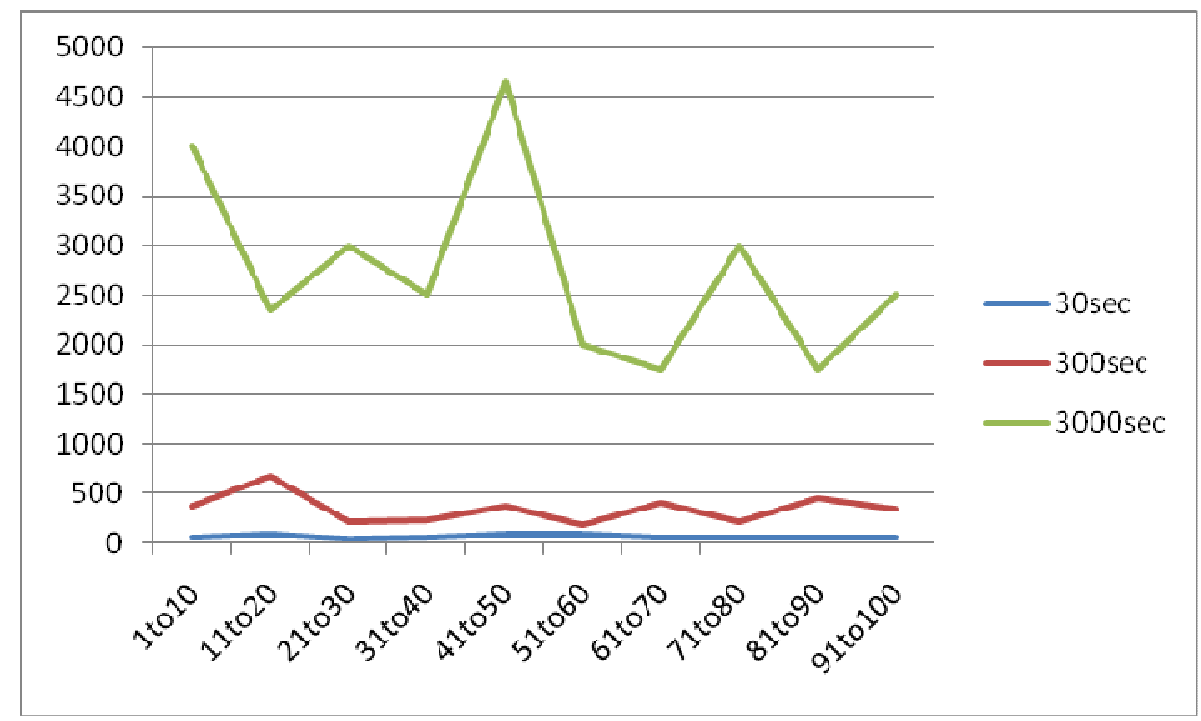

Figure 3. Packet Drop at MAC layer for OLSR.

LANMAR that is also a proactive routing protocol depicts the similar results as the earlier proactive routing protocol and of performs best in the case of smallest simulation time that is taken to be 3000 seconds. The highest packet drop witnessed at the MAC layer in this case of routing is about 3500 packets and the lowest being above 50 packets.

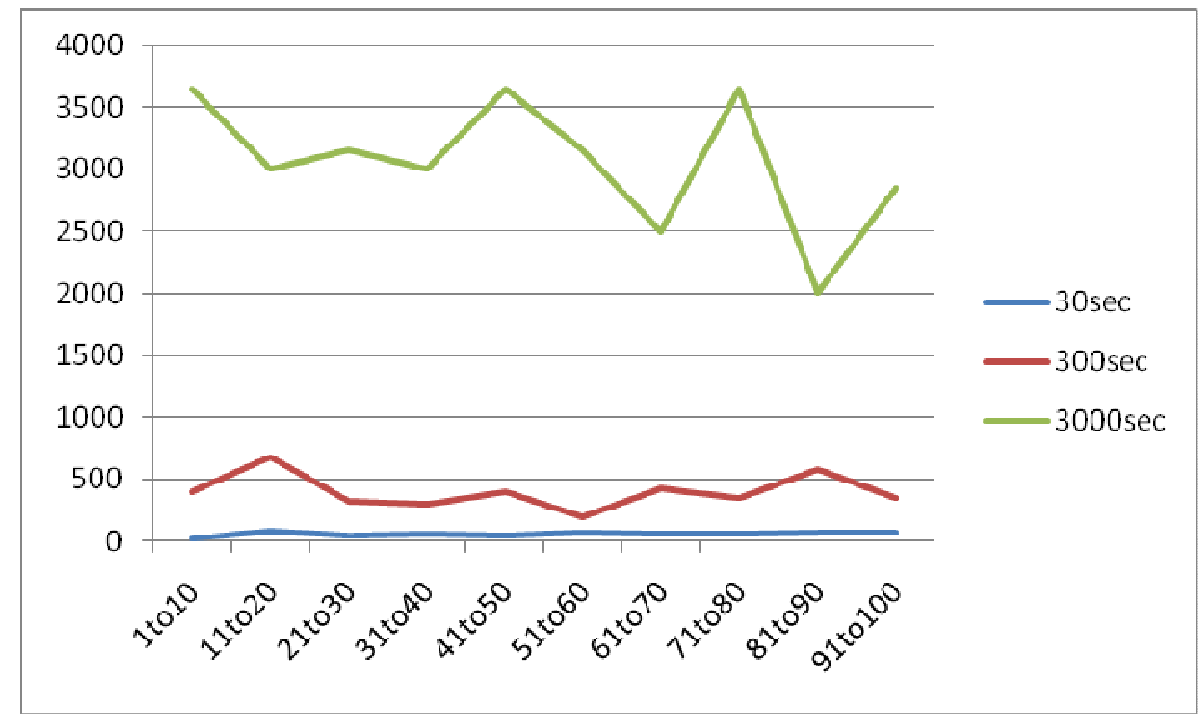

Figure 4. Packet Drop at MAC layer for LANMAR 
International Journal of Wireless \& Mobile Networks (IJWMN) Vol. 3, No. 6, December 2011

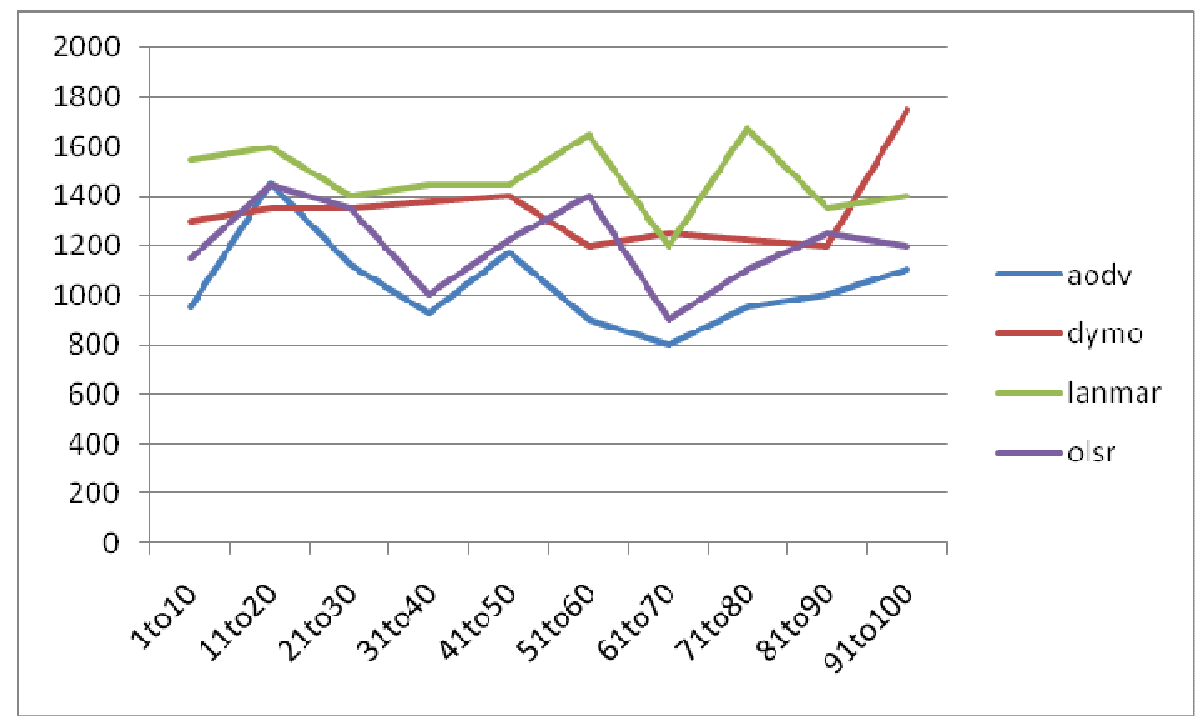

Figure 5. Packet Drop at MAC layer for First Scenario.

For the first scenario having the UGSs as well as the UGVs static the OLSR performs the best with the lowest number of packets dropped at the MAC layer and having the least high packet dropped value of 2900, and LANMAR being the least performing routing protocol with the highest packet drop rate of more than 5000.

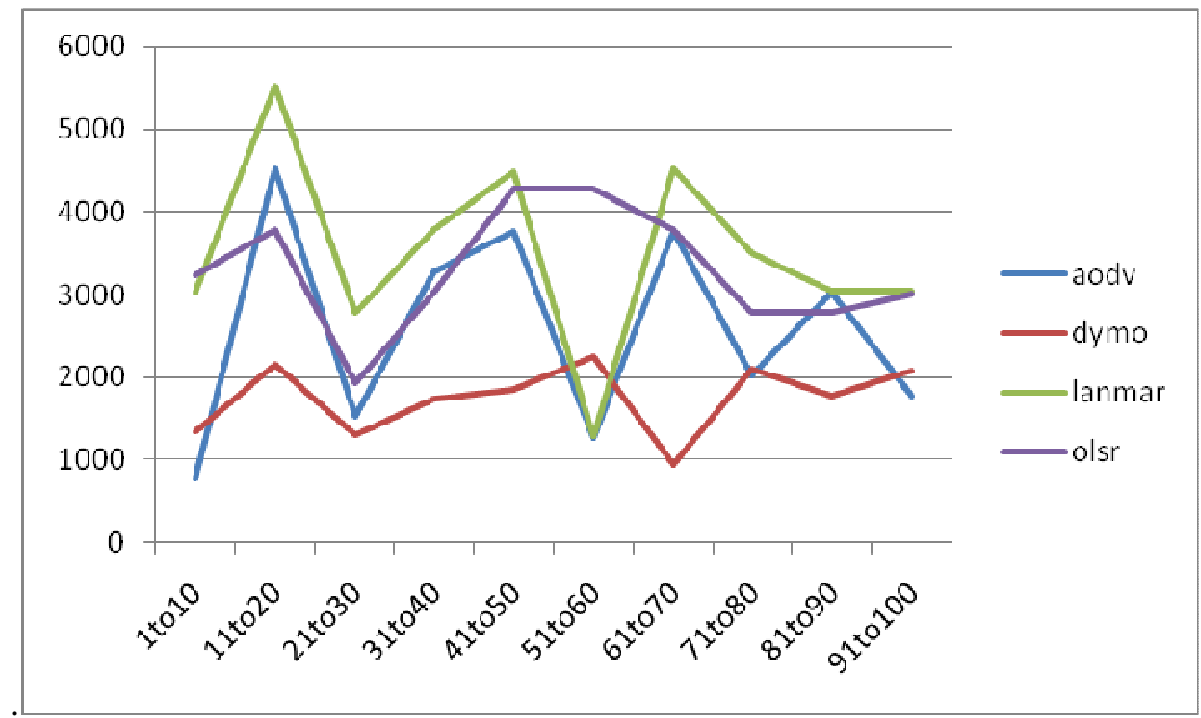

Figure 6. Packet Drop at MAC layer for Second Scenario.

In the case of second scenario having the UGSs as Static and UGVs mobile the total number of packets dropped is highest for the OLSR routing protocol having the packet drop rate more than 2900 and the best MAC Layer performance is depicted by the AODV having the lowest packet drop rate as below as 500 packets. 
International Journal of Wireless \& Mobile Networks (IJWMN) Vol. 3, No. 6, December 2011

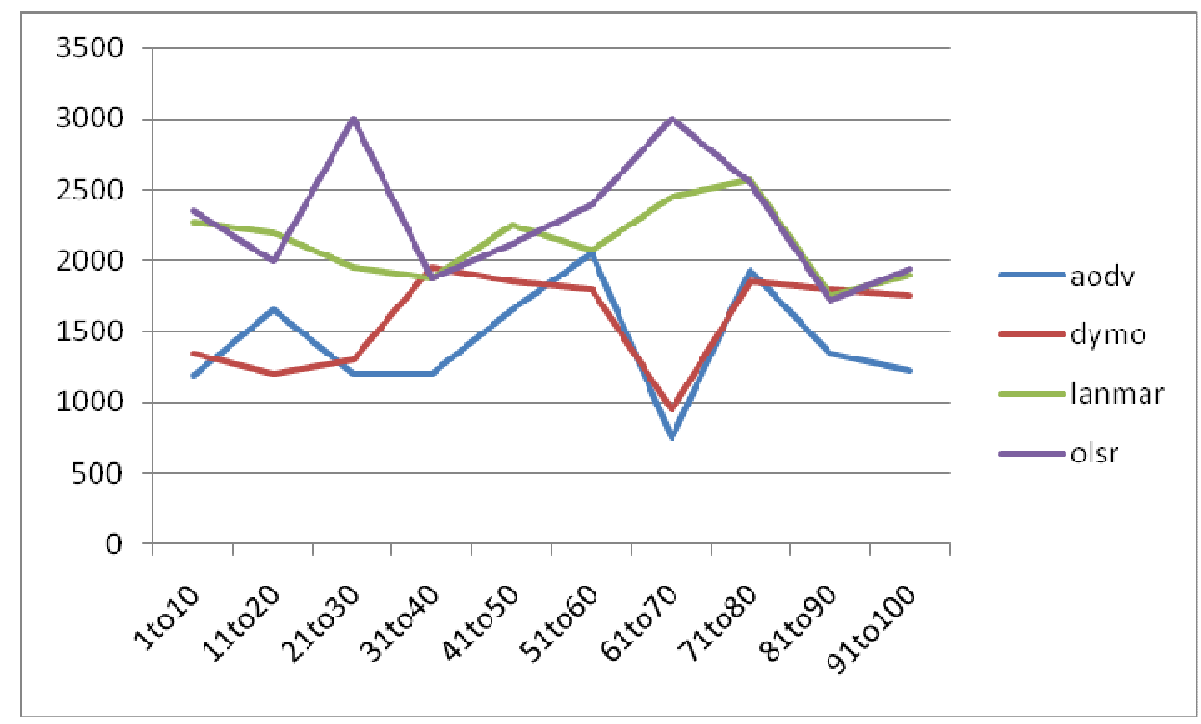

Figure 7. Packet Drop at MAC layer for Third Scenario.

For the third scenario having the UGSs as mobile and UGVs static the lowest packet drop rate has been depicted by the AODV protocol having average packet as low as 800 packets. While the highest packet drop rate has been witnessed in the case of OLSR having the highest packet drop rate of more than 2900 packets being dropped.

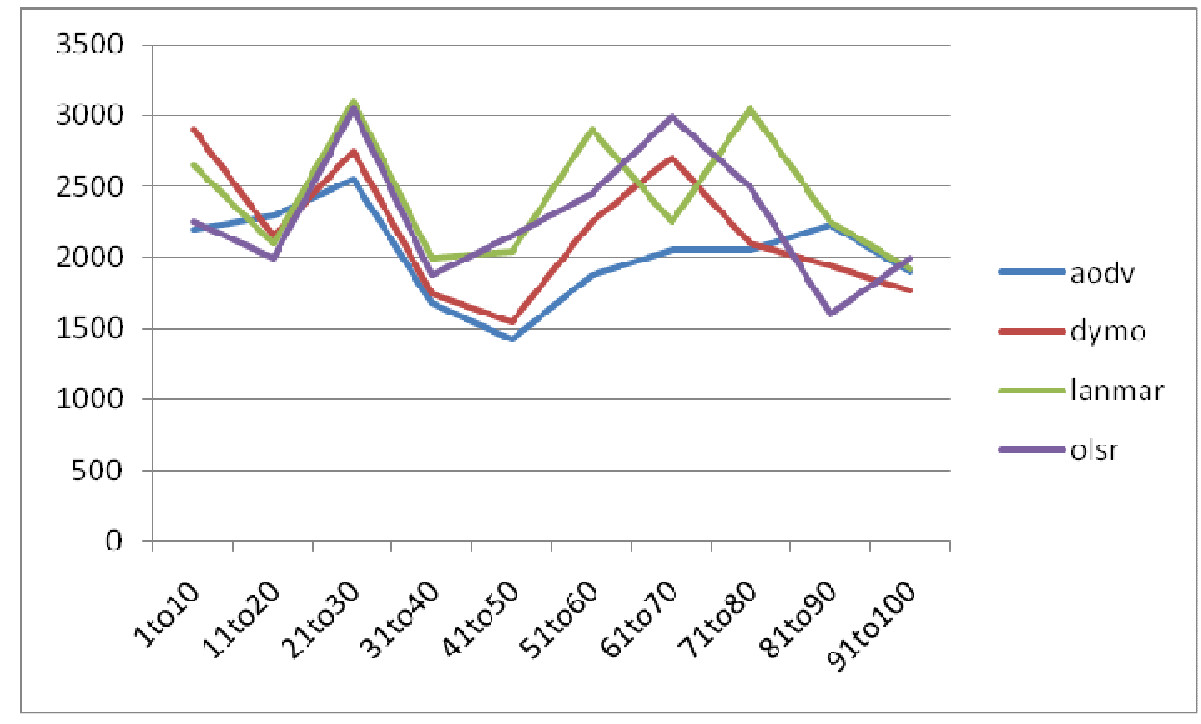

Figure 8. Packet Drop at MAC layer for Fourth Scenario

In the case of fourth scenario having both the UGSs as well as UGVs are mobile the AODV depicts the best performance with the lowest number of packets being dropped having the lowest packet drop value of 700 packets. While the worst performance is being depicted by the DYMO routing protocol in this scenario having the highest packet dropped value of 1700 .

Recent surveys in sensor network simulation can be categorized between less flexible but precise simulation based advance and more generic but less elaborated network simulator models. Simulator which furnishes a rich suite of following models: sensing stack to model 
wave and diffusion anchored sensor channels, a precise battery model, processor power use model, energy usage model and sensor network based traffic pattern. We also introduce our study on the effects of elaborated modeling on the functioning of higher layer protocols. We describe the affect of using precise models for battery, processor power usage and tracking models on the network layer stats as network lifetime and accessibility, throughput and routing operating cost. Our results show that comparative MAC layer packet drops at various time durations of simulation. Next section discusses the results for packets dropped at the MAC Layer when the two reactive and proactive protocols were implemented for various mobility condition based scenarios.

\section{CONClusion}

Designing a MAC protocol which can improve energy-efficiency to extend network lifetime in wireless sensor networks is a challenging problem. It is mainly due to stringent resource constraint both in sensor nodes and in wireless media. Several energy-efficient medium access control protocols both contention-based and reservation-based for the wireless sensor network that have been proposed by the researchers are presented in this paper. The design of an optimized MAC protocol for energy efficiency also depended on the actual application. However, no specific MAC protocol has been accepted as a standard. Another reason is the lack of standardization at lower layers (physical layer) and the sensor hardware. Therefore, it will be difficult to have one standard MAC protocol which will work for all possible WSN applications. Therefore, still a lot of work has to done in working out a MAC protocol which will adapt its behavior based on the applications. The research area of ad hoc and sensor network has very much attracted the academic domain as well as the industry both due to its wide-ranging possible application for anytime, anywhere, and any how communication scenarios.

This wide spectrum of applications possible for sensor networks has made the network vividly applicable and acceptable. The routing protocol for sensor networks has been a dynamic research area altogether through the present decade. Although wide efforts have been exercised so far on the routing problem in wireless communications, there are still some challenges via multicasting that still confront effective solutions to the routing problem. A number of such protocols have been purposed developed and implemented also. But no protocol has been found to be best for the wide domain of sensor network applications. Each protocol possesses its advantages and disadvantages. Counting the constraints followed by the networks the routing algorithms have been updated and modified time to time to make the routing more and more efficient and accurate. The present work proposes to find out the effect of different patterns of node mobility within the network. The results though don't present a steep comparative orientation of the results towards a specific routing protocol but the comparative study leads towards some interesting results.

Further research is needed to find most suitable protocol for each and every scenario condition so that an optimized routing protocol could be suggested for various real life applications have concurrency to the mentioned scenarios of the simulated wireless network environment.

\section{REFERENCES}

[1] C.K Toh, \& E.M.Royer, (Apr, 1999) "A review of current routing protocols for ad hoc mobile wireless network", IEEE Personal Communications 15, pp 46-55.

[2] I.F Akyildiz, Su. Weilian, Y.Sankarasubramaniam, \& E Cayirci, (2002) "A survey on sensor networks" Communications Magazine, IEEE Volume: 40 Issue:8 , pp 102-1148.

[3] C.Santivanez, B.,McDonald. I.,Stavrakakis,\& R.Ramanathan "Making link state routing scale for ad hoc network". DOI=10.1145/501417.501420. 
International Journal of Wireless \& Mobile Networks (IJWMN) Vol. 3, No. 6, December 2011

[4] S Basagni, M.Conti, S.Giordano, \& I.Stojmenovic (2004) "Mobile Ad Hoc Networking" IEEE Press and John Wiley and Sons, Inc., New York. ISBN 0-471-373133.

[5] C.Perkins, E.B.Royer, \& S Das, (2003) "Ad hoc on-demand distance vector (AODV) routing" RFC 3561.

[6] I.Chakeres, \& C.Perkins, (2008) "Dynamic MANET On-demand (DYMO) Routing”. IETF MANET, Internet-Draft, 5 December, draft-ietf-manet-dymo-16.

[7] Qualnet: http://www.scalablenetworks.com.

[8] W.,B Heinzeelman, A.Chandra Kasan, , \& H. Bala Krishnan (2000) "Energy-Efficient communication protocol for wireless micro-sensor networks." Proceedings of the 33rd Hawaii International Conference on System Sciences, pp. 3005-3014.

[9] C.Siva Rama, Murthy\& , B.S Manoj "Ad hoc Wireless Networks: Architecture and protocols,"Second edition, Prentice Hall.

[10] Sanatam Mohanty, \& Sarat Kumar Patra, (2010) "Performance Analysis of Quality of Service Parameters for IEEE 802.15.4 Star Topology using MANET routing” ACM 978-1-60558-812-4.

[11] Maneesh Varshney, \& Rajiv Bagrodia (2004) "Detailed models of sensor networks simulations and their impact on network performance" Proceeding of MSWiM '04 Proceedings of the 7th ACM international symposium on Modeling, analysis and simulation of wireless and mobile systems.

[12] P. F. Tsuchiya, (1988) "The Landmark Hierarchy: a new hierarchy for routing in very large networks", In Proceeding of SIGCOMM '88 Symposium proceedings on Communications architectures and protocols Issue 4 vol.18, pp 35-42.

[13] H. Ren, M. Q H.,.-Meng, \& X Chen (2006). "Investigating network optimization approaches in wireless sensor networks". In proceedings of International conference on Intelligent Robots and Systems, IEEE/RSJ, pp2015-2021.

[14] S.R. Das, R. Castaneda, J.Yan, \& R. Sengupta, (1998) "Comparative performance evaluation of routing protocols for mobile ad hoc networks" in Proceedings of $7^{\text {th }}$ international Conference on Computer Communications and Networks, pp153-161.

\section{Authors}

Ms. Manjusha Pandey is a $\mathrm{PhD}$ student in the Department of Information Technology at Indian Institute of Information Technology, Allahabad, India. Her Wireless Sensor Networks, Privacy in Wireless Communication, Privacy and security in Digital \& Mobile Communication, Signal Processing and Vehicular Technology. Contact her at rs58@iiita.ac.in

Dr. Shekhar Verma received his Ph.D. degree from IT, Banaras Hindu University, Varanasi, India in Computer Science and Engg. He is Associate Professor in Information Technology at Indian Institute of Information Technology, Allahabad, India. His research interest areas are Computer Networks, Wireless Sensor Networks, Vehicular Technology, Cryptography, Information and Network Security.
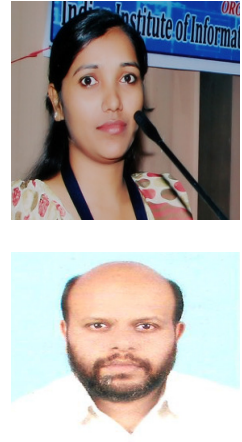